Letras, Lima 48 (84-85): 49-70, 1976.

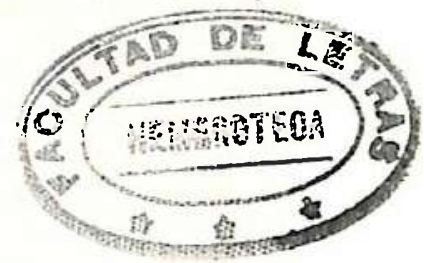

\title{
El concepto de realidad
}

\author{
FERNANDO BOBBIO ROSAS
}

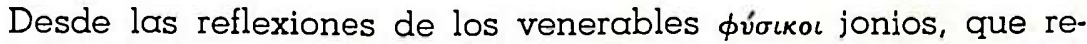

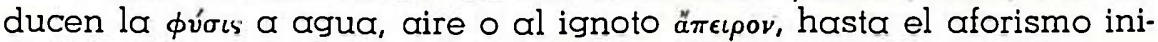
cial del Tractatus, y desde la vieja polémica entre los defensores

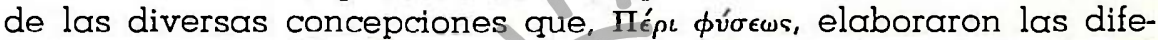
rentes escuelas filosóficas griegas, hasta los casi contemporáneos ataques de Moore a Bradley (1). los resultados logrados luego -y a través- de más de veinticinco siglos de meditación y debate, en lo que concierne al concepto de realidad, no son todo lo claro, preciso y terminantes que cabría esperar. Quizás, entre otros motivos, por la imposibilidad de definirla; al menos, en los sentidos más rigurosos del término, por ejemplo, qquél que exige que lo definido pertenezca ia una especie que a su vez, está incluida en algún género; pero, si lo que se intenta definir es la totalidad entonces, se trata de uña tarea imposible-der fealizar.

Que aquello de lo que intentamos dar un concepto -ya que no definir-, la realidad, es la totalidad de lo que existe, es un modo de decirlo; podemos, alternativamente, afirmar que todo lo que existe es la realidad (2). Formulaciones semejantes son todo lo que es, todo lo que hay; igualmente, el universo, el mundo, la na-

(1) Appearance and Reality de Bradley ha sido un estímulo importante de la producción filosófica de Moore. Limitándonos a los más específicos ataques de éste al idealismo de aquél podemos citar: "The Conception of Reality" y "External and Internal Relations" (Philosophical Studies) y los capítulos 11-12 y 15-16 de Some Main Problems of Philosophy.

(2) Buena parte de la tarea esclarecedora de Moore ha consistido en establecer esta sinonimia frente, por ejemplo, a ciertas afirmaciones bradleyanas sobre entidades que son algo, pero sin ser reales, o que son reales pero no existen (Cf. los lugares de la obra de Moore señalados en la nota anterior). 
turaleza (3), el ser. Pero todo esto no constituye ningún avance. La única posibilidad fructífera consiste en categorizar, clasificar, distinguir, diferenciar, dividir o separar esta realidad en sus elementos constitutivos, caso de tenerlos; pues, si fuese homogénea, no tendría elementos y, así, no habría nada que separar y muy poco de qué hablar.

Pero, el ser no es ni conceptual ni empíricamente homogéneo. Por consiguiente, puede ser $-\mathrm{Y}$ ha sido- categorizado. Y los esfuerzos por lograr una categorización lo más objetiva posible constituyen una buena parte de la historia de la filosofía; pero, la mayor parte de dichas clasificaciones ha sido meramente conceptual. Lo son, no solamente, la división del mundo en uno sensible y otro inteligible, o la que nos habla de res cogitans y res extensa, sino también la que señala como sus elementos los átomos y el vacío, - la que la reduce -como hace un monista contemporáneo-, a "la materia, sus propiedades y funciones" (4).

Lo que nos interesa $-\mathrm{y}$ nos proponemos presentar en este trabajo- es una clasificación de la realidad desde el punto de vista de nuestra experiencia sensible inmediata que es -no la percepción absolutamente pura, que parece que no existe, sinola que experimentamos corriente $y$ cotidianamente los que somos simples mortales sencillamente racionales y no filósofos racionalistas - o empiristas, o lo que sea-cargados con el lastre de más de dos milenios y medio de errores, prejuicios y confusiones filosóficas. Dicho de otro modo, tratemos de responder la pregunta: "¿qué percibimos, es decir, qué vemos, oímos, tocamos, palpamos, olemos y gustamos? . ll La primera respuesta, la más simple y directa es que percibimos las cosas que nos rodean. Vemos a las personas, sillas, mesas, árboles, gatos, etc. Oímos voces y pasos de personas, ladridos de perros, el ruido de los vehículos; sentimos la tiza o el bolígrafo entre las yemas de los dedos. Todo ello, personas, muebles, árboles, animales, vehículos, además de piedras, cuerpos celestes, etc. es lo que, en general, llamamos cosas. Luego, la realidad, que es lo que existe, lo que hay, lo que

(3) En EI concepto de la naturaleza (Madrid, Editorial Gredos, 1968), Whitehead indica, sin mayor explicación, que la realidad es algo más amplio y complejo que la naturaleza (p. 168). En Proceso y realidad (Buenos Aires, Editorial Losada, 1956), establece una distinción - ni muy clara, ni muy distinta - entre 'actual' y 'real' que podría dar Ia clave de la diferencia anterior que, por nuestra parte, consideramos inexistente. En Russell encontramos una identificación implícita entre realidad y mundo cuando dice: "Entiendo por realidad todo lo que habria de ser mencionado en una completa descripción del mundo" (Ĺógica $y$ conocimiento, Madrid, Taurus Ediciones, 1966, p. 314). Por supuesto que se guarda muy bien de aclarar qué sea ese "todo". 1964, p. 22. 
nos rodea y podemos percibir, se compone de cosas. Llegados a este punto podemos, si queremos, invocar la ayuda de la etimología; en efecto, nuestra palabra castellana 'realidad' proviene de la latina res cuya traducción española es 'cosa'. Más aún, el término cosa - que aún no hemos precisado- se aplica no sólo a las cosas mencionadas anteriormente, tales como personas, mesas y árboles, sino que también se suele usar para referirse a otro tipo de "cosas", como cuando una persona le dice a otra: "Le diré un par de cosas", o: "Ayer ocurrió una cosa muy divertida" (5).

Esto ha permitido que algunos filósofos afirmen que la realidad está constituida por las cosas y que las cosas son la realidad o, al menos, los elementos básicos o unidades irreductibles que la componen. Así, por ejemplo, Khatchadourian afirma que "objects are, in a basic sense, the "building blocks" of the physical world..."; aclarando un poco más adelante, "I use "object" to refer to perceived objects, following ordinary usage" (6). Bochenski, por su parte, nos dice que el "mundo consta de cosas (cosas, substancias), como montes, plantas, hombres, etc. que son determinados mediante diferentes propiedades, por ejemplo, colores, formas, aptitudes, etc., y que están vinculadas entre sí mediante relaciones. El nombre filosófico genérico para todo lo que puede ser, es el de ente (7).

Frente a esta posición otros filósofos sostienen que la realidad está compuesta por hechos -y los hechos son la realidad-. El ya mencionado aforismo inicial del Tractatus así lo establece:

\section{Die Welt ist alles, bwas der Fall ist.}

$Y$, por si la idea no estuviese ya claramente expresada, es ampliada de inmediato, rechazando, además, que la integran cosas; al menos, como los elementos básicos:

Die Welt is die Gesamtheit der Tatsachen, nicht der Dinge.

Martin se expresa de manera muy parecida cuando afirma que:

The totality of facts is everything that is the case... ( 8 ).

(5) John Hospers. Introducción al análisis filosófico. Buenos Aires, Editorial Macchi, 1966, Vol. I, p. 11.

(6) Haig Khatchadourian: "Objects and Qualities". En American Philosophical Quarterly. Philadelphia. Vol. 6. 1968, pp. 103 y 104. drid, Rialp, 1962 , p. 18.

(7) I. M. Bochenski. Los métodos actuales del pensamiento. Ma-

(8) R. M. Martin: "Facts: What They Are and What They Are Not". En American Philosophical Quarterly. Philadelphia. Vol. 4. 1967, p. 269. En la página siguiente leemos: "We need not comment at iength upon the niceties of Wittgenstein's use of "fact" in the Tractatus". Ni sólo en el Tractatus, ni tan solamente Wittgenstein. 
Dentro de esta misma posición Whitehead no es menos categórico:

Si hemos de buscar la substancia en alguna parte, yo la encontraría en los acontecimientos, que son en cierto sentido la última substancia de la naturaleza (9).

Russell se limita a señalar que

el mundo contiene hechos, que son lo que son, pensemos lo que pensemos acerca de ellos...

$\mathrm{y}$, ampliando la tautología anterior, agrega:

Cuando hablo de un "hecho" - no me propongo alcanzar una definición exacta, sino una explicación que les permita saber de qué estoy hablando- me refiero a aquello que hace verdadera o falsa una proposición.

Con lo que simplemente repite lo que ya había señalado un poco antes al señalar que los hechos son

aquello que determina la verdad o falsedad de una proposición, aconteciendo cuando nuestro enunciado es verdadero y dejando de acontecer cuando nuestro enunciado es falso (10).

Esta relación entre hechos y probosiciones es también resaltada por Martin, cuando dice que:

Facts are intimately linked with the semantic notion of factual or synthetic truth, not just with that of truth. Nonetheless facts are not just factual truths (11).

Partiendo de esta relación, existente y evidente, algunos filósofos dan un paso más y llegan a identificar hechos y proposicio. nes, como lo hace Carnap, que menciona a Ducasse quien

identifies facts with true propositions....

àgregando que él (Carnap) está

inclined to think, like Ducasse that it would not deviate too

(9) A. N. Whitehead. El concepto de naturaleza, p. 30 . Como veremos más adelante, esto no es muy coherente con otras afirmaciones contenidas en esta misma obra.

(10) Bertrand Russell. Lógica y conocimiento, pp. 285 y 269.

(11) Op. cit., p. 276. 
much from customary usage if we were to explicate the term 'fact' as referring to a certain kind of proposition [...] What properties must a proposition have to be a fact...? First, it must, of course, be true; second, it must be contingent (or factual); thus it must be Ftrue. I think that still another requirement should be added: The proposition must be specific or complete in a certain sense; but I am not sure what degree of completeness should be required (12).

El anterior es el mismo punto de vista de Fitch quien afirma facts themselves are nothing more or less than true propositions.

Y agrega:

False propositions are merely those propositions which are not facts.

Y basándose en esta identificación llega a su tesis radicalmente gramatical cuando dice que

all entities are reducible to propositions, which remains as the final irreducible realities. There seems to be no other single type of entity to which such a reduction of all other types of entity can be made. Hence no other entities qualify as ultimate realities in the same degree as propositions.

Pero no solamente lás ủltimas, cino, quizá, las únicas realidades, como ha manifestado un poco antes

all entities are reducible ultimately to propositions, so [...] propositions are the final realities and indeed the only realities (13).

Dejando de lado esta última posición, hasta aquí hemos visto que, para unos filósofos, los elementos fundamentales de la realidad son las cosas mientras que, para otros, dichos constituyen-

(12) Rudolf Carnap. Meaning and Necessity. Chicago, University of Chicago Press, 1960, p. 28.

(13) F. B. Fitch: "Propositions as the Only Realities". En American Philosophical Quarterly. Philadelphia, Vol. 8. 1971, pp. 100, 103 y 99. Además, sobre "Hechos y proposiciones", pueden también verse los ensayos de Ramsey y Moore presentados y publicados por la Aristotelian Society. Reimpresos en Los fundamentos de la matemática y otros ensayos y en-Philosophical Papers, respectivamente. 
tes básicos, primitivos o irreductibles son los hechos. Creemos que argumentar en favor de una $u$ otra postura es embarcarse en una discusión bizantina, o casi. Tanto cosas como hechos son lo que se nos presenta primaria -y palmariamente- ante nuestra percepción; y la tarea más importante consiste en demarcar, hasta donde sea posible, cada una de estas dos clases en que estamos dividiendo la realidad; es decir, caracterizar de la manera más completa $\longrightarrow$ menos incompleta- los conceptos de cosa y de hecho.

¿Qué es una cosa? En primer lugar, establezcamos ciertas precisiones terminológicas. Se suelen tomar como expresiones sinónimas $\longrightarrow$ casi-, 'ente' y 'objeto'. Pero, cuando se hace esto se les añade sobre todo a la última, $-\mathrm{y}$ probablemente con la finalidad de lograr una mayor claridad y precisión- algunos adjetivos, tales como 'material', 'físico' o 'perceptible' -lo que está contrarrestado por el agregado de otros adjetivos menos convenientes, o totalmente inconvenientes- (14).

'Ente' es, como señala Bochenski - citado más arriba-, todo lo existente. 'Objeto' es un concepto de otro tipo, es un concepto relacional, para que existan objetos se requiere de un sujeto; no hay objetos sin sujeto; es, pues, producto de la relación entre un sujeto y su entorno, su ambiente, su mundo, la porción de realidad que más inmediatamente lo rodea y que el sujeto objetiviza. Por lo mismo, todo y nada es objeto. Nada, nada de lo existente, es decir, ninguna cos ni ningún hecho es un objeto; y todo, todos los hechos y todas las cosas pueden ser y son objetos; pero, objetos de, de la percepción de la atención, del recuerdo, de los sentimientos de algún sujeto que, por su parte, también puede ser objetivado por otro "sujeto. P Porilo mismo, ecuando Khatchadourian - citado más arriba- señala que con 'objeto' se refiere a 'objetos percibidos', no está enunciando una tautología, pero tampoco está proporcionando mucha información. Y, además, aunque se está refiriendo a cosas, como quedará aclarado más adelante (15), dicha caracterización - si podemos llamarla así-, no es nada es-

(14) Mientras unos filósofos se han empeñado en aclarar y simplificar - hasta el punto más conveniente- el panorama conceptual y terminológico, otros se han dedicado, con el mismo o mayor entusiasmo, a complicarlo, confundirlo y oscurecerlo. Entre éstos tenemos, entre otros, a aquéllos que añaden adjetivos incompatibles a ciertos sustantivos; por ejemplo, algunos de los adjetivos que, tradicionalmente, acompañan a 'cosa' o' 'ente', tal como lo señala Max Hocutt ("What We Perceive". En American Philosophical Quarterly, Philadelphia, Vol. 5, 1968 , p. 48): "They are no inexisting things or subsisting things. Such things are not kinds of things and, therefore, they are not things". El absurdo de hablar de "Cosas" inexistentes es llamado por Quine "a certain verbal perversity" (Word and object, Massachusetts, The MIT Press, 1965, p. 3).

(15) Haig Khatchadourian. Op. cit., pp. 104 y 110. 
pecífica, porque objetivamos toda la realidad, que de otro modo sería incognoscible. Y cuando, más adelante, en el mismo artículo de Khatchadourian, encontramos que afirma que hay cosas que no son objetos,

buildings, hills, mountains, [...] are individual things, though not objects in the relevant ordinary sense of the word....,

simplemente no lo entendemos. Edificios y montes, entre otras cosas, son objeto de nuestra percepción, de nuestro agrado -o desagrado-, de nuestro recuerdo, etc. Además, y justamente con relación a los edificios podemos hacer extensivo lo que, en otro lugar y refiriéndose a mesas, señala el mismo autor, cuando contrasta cosas - lo que llama objetos- con hechos,

it makes no sense -in contrast to events, occurrences, happenings, processes and activities - to say such things as: "This (object) started at 10 a.m. yesterday and ended at 11 p.m. this morning"; though we say: "This table was made on July 15,1966 and was dropped to pieces on June 4, 1967". Objects come into existence; and some do so by being produced (16).

Igualmente, la afirmación de Quine:

Physical thing generally, however remote, become known to us only through the effects which they help to induce at our sensory surfaces...

reafirmada un poco más dadelante:

The proposition that external things are ultimately to be known only through their action on our bodies should be taken as one among various coordinate truths, [...] about [...] physycal things... (17)

tampoco es muy específica. No es propiedad exclusiva de las cosas impresionar nuestros sentidos. Que los hechos también se perciben es algo de lo cual se percata incluso el perspicaz Russell:

No se cree en hechos. Se percibe hechos (18).

'Objeto' no es, pues, sinónimo de 'cosa'; una cosa es un objeto cuando alguien la convierte en tal; pero, esto puede ocurrir con todo lo que existe; luego, afirmar que una cosa es un objeto es, en la práctica, no decir nada. Con lo que podemos empezar a

(16). 'Op. cit., pp. 114 y 110.

(17) Quine. Op. cit., pp. 1 y 4.

(18) Bertrand Russell. Op. cit., p. 312. 
tratar de averiguar qué es una cosa. Hay varias respuestas posibles, parciales y aproximativas:

Una cosa, en sentido cotidiano, está constituida por un manojo de cualidades sensibles que pertenecen a varios sentidos diferentes, pero que se supone coexisten todas en una porción continua de espacio (19). The first property, then, by means of which I propose to define a material object is that it must have position in space (20).

Pero en ninguna de estas dos formulaciones se señala lo fundamental del concepto en cuestión que es que una cosa es

something relatively "stable" or "enduring"... (21)

Whitehead también pone el énfasis en esta última característica:

Los factores de la naturaleza que carecen de la condición de paso serán llamados objetos.

Y, luego:

Los objetos son los elementos de la naturaleza que no pasan.

Y como ejemplo de cosa, estable y permanente, una de las más duraderas que se puedan presentar, menciona a la pirámide, lo que le sirve, al mirmo tiempo, para poner de relieve, contrastando, la diferencia entre hechos -que pasan-y cosas -que quedan- (22):

Un objeto es uñna entidadidelotroltiposque la de un acontecimiento. Por ejemplo el acontecimiento que es la vida de la naturaleza en la Gran Pirámide ayer y hoy es divisible en dos partes, a saber: la Gran Pirámide ayer y la Gran Pirámide hoy. Pero el objeto reconocible que se llama la Gran Pirámide es el mismo objeto hoy que ayer.

$Y$, casi enseguida pasa a remarcar la intemporalidad de las cosas:

Un objeto se halla en cierto sentido fuera del tiempo. Se ha-

(19) Ôp. cit., p. 153.

(20) G. E. Moore. Some Main Problems of Philosophy. New York, Collier Books, 1966, p. 145. Además propone, para otorgar a algo el status de cosa -objetos materiales, dice él-, otros dos requisitos: "The first negative property is this: I propose that no sense-datum, or part of a sense-datum, or collection of sense-data, is a material object or a part of one. [...] And the second [...] is this: That no mind, and no act or conciousness can be a material object" (Op. cit., pp. 145 y 147).

(21) Haig Khatchadourian. Op. cit., p. 110.

(22) El concepto de naturaleza, pp. 141 y 160. 
lla en el tiempo únicamente de una manera derivada, por tener relación con los acontecimientos que llamo "situación" (23).

Resumiendo lo expuesto hasta aquí, tenemos que las cosas ocupan un espacio determinado y tienen cierta permanencia -implícita en el "ocupar"- - Pero, hay algo más, que es lo que podríamos llamar la individualidad de la cosa. Una cosa es una unidad que destaca, o se puede distinguir, o individualizar, dentro y de entre un fondo, -el que constituye el panorama total de nuestra percepción del momento-; y, además, el carácter de unidad de la cosa varía -o puede hacerlo- 1) con las circunstancias que a ella misma le conciernen (24), 2) según lo establezcan ciertas convenciones lingüísticas (25), y 3 ) según los intereses,

(23) Op. cit., pp. 91-92. En su Proceso y realidad, p. 288, nos ofrece esta otra versión del significado de cosa: "cosa" -o su equivalente "entidad" - no significa más que el ser uno de los "muchos" que encuentran sus habitáculos en cada caso de concrescencia. Cada caso de concrescencia es él mismo la cosa individual nueva en cuestión".

(24) Haig Khatchadourian. Op. cit., p. 112:

A chair as a whole is, at a given moment, in a certain place, legs and all. Now suppose we detach one leg, but keep it where it was before it was detached. It can now be regarded as a separate object and can (therefore) be said to occupy a place of its own slightly different from the place occupied by the (rest of the) chair. If, finally, we imagine the detached leg glued back to the chair, at the point at which it originally was glued, we would stop talking of it as occupying a place of its own, and speak only of the chair as a whole - legs and all- occupying a certain place. (25) Op. cit., p. 115:

linguistic convention plays an important rol in determining whether some occupant of space is (to be regarded as) part of an object but not also an object in its own right, an object, a collection of objects, or some kind of complex of objects. Thus what we call a cagon is, conventionally, both a thing in the ordinary meaning of "thing" (an object in our extended use of "object"), and part of what we call a train; which is (also) ordinarili regarded as a thing ("object")... Generally speaking," something... which may form part of a larger thing by being spatio-temporally connected to it in the same manner, is conventionally regarded both os an individual thing and part of a larger or more complex thing.

El condicionante lingüístico, que menciona Khatchadourian, es más agudamente resaltado en el siguiente pasaje de otro autor en el que se sostiene que el lenguaje influye hasta en nuestras más básicas y, aparentemente, neutras relaciones con la realidad: "La cualidad y estructura de las sensaciones (percepciones), o al menos la cualidad y estructura de aquellas sensaciones que entran en el campo de la ciencia son independientes de su expresión lingüística. Dudo mucho acerca de la validez, incluso aproximada, de este supuesto" (P. A. Feyerabend, Contra el método, Barcelona, Ediciones Ariel, 1974, p. 59). 
variables, del hablante en la situación y condiciones en que se encuentre.

El que las cosas "duren" no significa, ni mucho menos, que sean eternas, ni tampoco inmutables $\mathrm{y}, \mathrm{nl}$ siquiera, que estén inmóviles; nada, absolutamente nada, lo está, excepto en el sentido limitado, relativo $\theta$ impreciso que tienen en el lenguaje cotidiano estos términos y todos los demás relacionados con ellos, tales como permanencia, constancia, estatismo, estabilidad, persistencia, continuidad, perdurabilidad, invariabilidad, inalterabilidad, y quizás otros que en este momento se nos escapan. La pirámide que utiliza Whitehead como paradigma de durabilidad también se está desgastando cada hora, cada minuto, cada segundo y cada inimaginable fracción de segundo que transcurre. También ella, como todas las cosas, desaparecerá en algún momento.

$Y$ esto nos conduce a otro problema, ¿cuándo la pirámide dejará de serlo -y cuándo empieza a ser una pirómide-? Tomemos un ejemplo más familiar, la tiza que vamos a utllizar es una cosa, Indiscutiblemente; luego de un rato - ¿qué es un "rato"?-. nos queda sólo un fragmento que, también, podemos considerar una cosa. Pero, en qué momento del tránsito de la tiza -que es un pedazo compacto de partículas de yeso- a la multitud dispersa de partículas de yeso, deja de ser una tiza, deja de ser una cosa. ¿Podríamos decir que cade partícula casi imperceptible -o imperceptible- de yeso es una cosa? Las moléculas, los átomos - las llamadas partículas elementales, ¿son cosas? ¿Dónde empieza y dónde termina "algo" de ser una cosa? Llegados a este punto lo único que podemos hacer es recordar lo que, líneas arriba, hemos señalado al tratar de la "individualidad" de la cosa (26).

Un problema simnlar surge con relación a' los límites, también relativos, es decir, imprecisos y fluctuantes, temporales de la cosa. Russell dice:

(26) No olvidemos de que estamos esbozando una categorización de la realidad desde el punto de vista de nuestra percepción, que no sólo es el modo más natural y directo de conocer sino, lo más importante, el fundamental. Así tenemos que si bien la ciencia postula la existencia de algunas entidades transempíricas, ello se hace a partir de los efectos observables que dichos entes provocan - o se les hace provocar-. Y cuando dicha postulación se debe, exclusiva o casi exclusivamente, a exigencias formales, no olvidemos que, primero, el contexto teórico general dentro del que se efectúa dicha postulación se ha elaborado a partir de bases observables $y$, segundo, que siempre se busca que la entidad postulada dé pruebas de su existencia, so pena de ser degradada - conceptualmente a mera fícción, como lo señala L. M. Lederman, a propósito del neutrino: "Obviously a particle that reacted with nothing whatever could never be detected. It would be a fiction. The neutrino is just barely a fact" ("The -Two Neutrino Experiment", In Solentific American, New York, Vol. 208, No. 3. 1963, p. 60).

(27) Bertrand Russell. Op. cit., p. 234

58 
Las cosas tienen a mi juicio una duración finita, cuestión de unos segundos, o minutos, o algo por el estilo (27).

Las cosas, independientemente del juicio de Russell, tienen, como todo lo que existe, "una duración finita" y esto ocurre desde la "vida" inconcebiblemente corta de las partículas elementales - sin que nos estemos pronunciando acerca de si merecen o no el calificativo de cosas-, hasta la de la estrella de más larga existencia posible, pasando por la pirámide del ejemplo whiteheadiano - probablemente la cosa creada por el hombre de mayor perduración-, todo tiene la mencionada duración finita.

Pero, y aquí podemos enlazar ésta con la dificultad anterior, pareciera ser que no estamos tentados a otorgar la categoría de cosa, al menos no tan fácilmente, a lo que más que durar, o estar, pasa; a menos, por supuesto, que querramos llamar cosa a todo lo existente, "cosa" que, como ya hemos visto, es posible. Una piramide es una cosa, una silla también lo es $e$, incluso, un castillo de arena. Pero, ¿lo son las partículas de yeso en que se disgregó la tiza,... los son las moléculas,... y los átomos? Nos resistimos a considerar como cosas a todo aquello cuya existencia es más bien un paso, un tránsito. Recordemos a Whitehead, -ya citado-:

Los factores de la naturaleza que carecen de la condición de paso serán llamados objetos.

Los objetos son losi elementos de la naturaleza que no pasan. No pasan para nuestra percepción; Y dentro de la relatividad del concepto -y del término- empirico podemos hablar de una permanencia -aún más- relativa, que va de la de la tiza que gastamos y el cigarrillo que consumimos a la de la silla que menciona Khatchadourian, -citado líneas arriba-, cuyos límites temporales señala, a la de la permanencia -relativamente- absoluta de la pirámide, a cuya vera podríamos pasar toda la extensión de nuestra corta vida sin apreciar el más mínimo cambio. Pero, por supuesto que todo pasa $-\mathrm{y}$ lo sabemos, y también lo supo Whitehead, y probablemente de un modo mucho más completo y profundo que cualquiera de nosotros (28) y si bien ello es más visible en los seres vivos,

(28) Podría parecer tonto tener que formular una afirmación semejante; pero cuando en nuestros más altos niveles educativos hay quienes creen ciega y sinceramente que el principio de identidad significa: todo objeto $\mathrm{y}$ todo concepto son siempre iguales así mismos [...] malentendiendo el 'siempre' en el sentido de que son inmutables; y que el principio de no-contradicción pro- 


\section{(O douleur! o douleurl Le temps mange la vie,...)}

el padre Cronos también se alimente de las cosas no vivas, las inmóviles, las cosas in-animadas, es decir, carentes de un ánima que las mueva, que las anime.

Lo anteriormente expuesto nos lleva a establecer una íntima relación entre duración y extensión, se trata de dos magnitudes que, corrientemente, son directamente proporcionales. (Con la adición de la propiedad de la densidad de los cuerpos quizá podría incluso establecerse alguna relación cuantitativamente determinable). Nos servimos de ambas magnitudes para establecer lo que es una cosa; pero, sin que exista ningún límite preciso, natural y objetivo entre las cosas y lo que no lo son.

Finalmente, un problema casi tan viejo como la misma filosofía, ¿cuándo una cosa se transforma en otra, cuándo una silla se convierte en un trasto inútil, cuándo un hombre se vuelve calvo, cuándo deja de ser adulto y empieza a ser un anciano? Russell intenta responder señalando que una cosa - la silla, el hombre-, continúa siendo esa misma cosa - 0 , mejor, continúa perteneciendo a la clase de cosas que llamamos sillas u hombres y no, todavía no, a la de las cosas que denominamos trastos o viejos-,

mientras no modifique su apariencia lo bastante como para que usted contase con un dato sensible diferente (29).

Pero aquí, igual que en el caso anterior, tampoco hay ningún límite preciso, naturab ni objetivo.

Pasando a ocuparnos de los heghos la primera cuestión que se presenta es la de tratar de caracterizarlos: Partamos, tal como lo hicimos en el caso anterior, de dar algunas palabras que -con cierta flexibilidad- pueden ser consideradas sinónimas de 'hecho'. Estas son 'acaecimiento', 'acontecimiento' - la palabra que prefiere emplear Whitehead, cuando se ocupa del tema-, 'evento', 'fenómeno' -a pesar del uso totalmente diferente que le dio Kant

clama: "A no puede ser simultáneamente A y no-A", es decir, otra vez la afirmación de que el objeto siempre posee sólo una cualidad (Iudin y Rosental, Diccionario de filosofía y sociología marxista, Buenos Aires, Séneca, 1939, p. 61; el subrayado es nuestro y nos releva de cualquier comentario), y que Aristóteles - de quien Farrington, uno de los poquísimos historiadores de la ciencia eruditos, inteligentes y de tendencia marxista, tiene que admitir que "fue el hombre que, individualmente, más hizo por el desarrollo de la biología"-, o no se dio cuenta de semejantes absurdos o bien, a sabiendas, "inventó" estas falsedades -y otras más- de puro reaccionario y servil, entonces hay que aclarar al máximo todas las afirmaciones que se formulen.

(29) Bertrand Russell. Op. cit., p. 285. 
se la emplea en el lenguaje, tanto corriente como científico, como sinónimo de hecho; así, se suele hablar de fenómenos físicos o naturales, de fenómenos psicológicos, sociológicos, etc.-, 'ocurrencia' y 'suceso'. Un concepto un poco más complejo es el que nos da la palabra 'proceso', sobre el que volveremos más adelante.

La idea fundamental contenida en todos estos términos, como puede apreciarse fácilmente, es la de cambio, tránsito, paso, fluencia, movilidad, devenir, dinamismo. Y este matiz es más notorio en unos que en otros, como por ejemplo, en suceso, ocurrencia, acontecimiento y acaecimiento, todos ellos sustantivos que evocan, inmediatamente, el verbo correspondiente. 'Hecho' conlleva más bien cierto matiz de congelamiento, de concrescencia y lo mismo sucede con 'fenómeno', sobre todo si se hace hincapié, como lo hizo Kant, en su etimología. 'Evento' también está en la misma situación -aunque algunos opinen exactamente lo contrario- (30),

(30) Charles Landesman: "Actions as Universal: An Inquiry into the Metaphysics of Action", En American Philosophical Quarterly, Philadelphia, Vol. 6, 1969, p. 250: "Actions are species of events, and events a species of attributes". C. E. M. Joad, Guia de la filosofía, Buenos Aires, Editorial Losada, 1940, p. 181: "Un suceso puede definirse más o menos como un acontecimiento en un momento dado, por ejemplo, el resplandor del relámpago, el disparo de un fusil, la erupción de un volcán". S. Körner, ¿Qué es filosofía?, Barcelona, Ediciones Ariel, 1976, pp. 153-154:

para las conjunciones de rasgos distintivos que cambian o son susceptibles de cambio y que pueden caracterizar una región particular del espacio durante un determinado intervalo de tiempo [...] emplearé el término "Evento". (con E mayúscula). Este término abarca no sólo aconteeimientos en el sentido más restringido del término, por ejemplo el estallido del trueno, en el que cambian con relativa rapidez una o más características de una región del espacio, sino también estados de cosas (situaciones), cuyas características son relativamente estables, procesos..., etc. Todo aquello incapaz de caracterizar una región particular del espacio durante un determinado intervalo de tiempo no es un evento. Las verdades matemáticas, las leyes de la naturaleza, los principios morales no describen eventos dada su intemporalidad.

Fitch, Op. cit., pp. 99-100:

An event occurs whereas a fact simply is [...] the event is in some location in spatio-time [...] occurrence emphazises this quality of posessing spatio-temporal location, a quality that events have but that some facts, for example, mathematical facts do not have. The facts that do have spatio-temporal location are ones that I call events. Thus historical facts can equally well be called historical exents, but mathematical facts cannot be called events.

En las cuatro citas precedentes hemos querido mostrar dos puntos; el primero, es la falta de deseos de precisar los términos; y, el segundo, la confusión y el desacuerdo que reina entre los especialistas; así tene- 
tonto en lo que se refiere a su etimología como a su uso, (por lo demás, no es una de las palabras más empleadas, al menos en castellono).

Esta íntima relación entre los hechos y el devenir, el transcurrir del tiempo -o, simplemente, el tiempo- es resaltada por Whitehead en una serie de pasajes de El concepto de naturaleza:

Hay tiempo porque hay acontecimientos y fuera de los acontecimientos no hay nada. [...] el espacio y el tiempo son en sí mismo abstracciones de los acontecimientos. No puede darse tiempo separado del espacio, ni espacio separado del tiempo, ni espacio ni tiempo separados del paso de los acontecimientos de la naturaleza. [...] el espacio y el tiempo no son sino modos de expresar ciertas verdades acerca de las relaciones entre los acontecimientos (31).

El término 'proceso' -ya mencionado- evoca un concepto algo más complejo, aunque podría reducirse al de hecho, lo que implica aceptar la existencia de varios tipos de hechos 0 , mejor dicho, las clasificaciones que hacemos de los hechos. Dentro de éstas podemos citar la de Russell; éste divide los hechos en particulares y generales (32), en simples - o atómicos- y complejos -o moleculares- (33), y -el colmo de la imaginación- hasta en

mos que para uno los eventos son atributos y para otro son ocurrencias; alguien afirma que los sucesos, por ejemplo, los relámpagos, son especies de acontecimientos, mientras que algún otro sostiene que los acontecimientos, por ejemplo, losctruenos, sonespecies de eventos. Aparte de todo lo anterior habría que remarcar que nada existe fuera del tiempo y del espacio; pese a Platón, a Fitch, a Körner o a la Reina de Corazones.

(31) A. N. Whitehead. Op. cit., pp. 79, 92, 158 y 187. Bunge también reconoce esto cuando escribe que "el tiempo, lejos de tener virtudes productivas y de "fluir" per se con prescindencia de la sucesión de los acontecimientos no es más que el ritmo de los procesos, el orden de los sucesivos (Leibniz)" (Causalidad. El principio de causalidad en la ciencia moderna, Buenos Aires, Editorial Universitaria de Buenos Aires, 1972, pp. 89-90). Extremando la nota, para el Heráclito contemporáneo

el universo pertenece al [...] torrente del cambio o "devenir" [...] (desde que la realidad jamás es una cosa sino que siempre está en vías de devenir otra) [...] resulta imposible descubrir cualquier cosa que pase por cambios pero que sea algo distinto de los cambios que le ocurren. En otras palabras, el universo mismo es un torrente de cambio perpetuo (C. E. M. Joad, Op. cit., p. 158).

(32) Bertrand Russell. Op. cit., pp. 258-259.

(33)

Op. cit., p. 287. 
positivos y negativos (34). Dentro de esta clasificación, o una similar, 'proceso' podría ser sinónimo de 'hecho complejo'; pero es un término muy usado y tiene un núcleo significativo propio.

En efecto, cuando hablamos de proceso lo hacemos para referirnos a una sucesión más o menos ordenada -aunque el orden lo pongamos nosotros, los hechos simplemente se dan- y más o menos prolongada de hechos. Y aquí volvemos a tropezar con la vaguedad e imprecisión del lenguaje; la palabra 'proceso' no está claramente delimitada. Se la emplea para designar desde el -tan simple y tan breve proceso de la combustión de una cerilla,

(34) Op. cit., p. 258, aunque luego se desdice en otros dos lugares de la misma obra de modo implícito $y$, a lo que parece, sin percatarse de ello; así, cuando en la página 92 afirma: "No hay hechos falsos, de ahí que no contemos con un hecho para cada proposición, sino tan sólo con un hecho para cada par de proposiciones". Lo cual es casi cierto; es decir, si nos restringimos al campo de la lógica bivalente y no incursionamos en la modal, por ejemplo. Pero, aparte de ello, el pasaje que acabamos de citar, está en contradicción con este otro (Op. cit., p. 296):

¿hay hechos negativos? ¿Hay algún género de hechos en que poder clasificar hechos como "Sócrates no está vivo"? [...] hay hechos negativos, es decir, [...] si, por ejemplo, ustedes dicen "Sócrates no está vivo", ha de darse un hecho que en el mundo real se corresponda con su proposición: el hecho de que Sócrates no vive.

Lo cual, a su vez, estálen contradicción con este otro pasaje de la misma obra, pp. 262-263:

Supóngase que es un hecho que Sócrates está muerto. Tienen ustedes dos proposiciones: "Sócrates está muerto" y "Sócrates no está muerto". Y ambas proposiciones corresponden a un mismo hecho: hay un solo hecho en el mundo que hace a la una verdadera y falsa a la otra. A cada hecho le corresponden dos proposiciones, la una verdadera y la otra falsa.

Lo más provechoso de todas estas divagaciones es que nos permiten darnos cuenta - una vez más- de la tremenda -y no sentida - influencia del lenguaje sobre el pensamiento de quienes lo empleamos. En efecto, es evidente que nuestro buen filósofo confunde hechos con proposiciones. $Y$, aunque a veces vislumbre esto, $y$ trate de evitarlo, como cuando "duda" de que haya hechos de la forma sujeto-predicado" (Op. cit., pp. 401-402); sin embargo, en otras ocasiones la mencionada influencia del lenguaje sobre sus categorizaciones lo lleva al absurdo, como cuando afirma que las tautologias son "hechos negativos": "Lo que es redondo no es cuadrado. $Y$ esto ha de constituir un hecho, $[\ldots]$ negativo" (Op. cit., p. 404). Su misma clasificación de los hechos es gramatical; aunque en su descargo habría que señalar que quizá no sea posible una clasificación absolutamente objetiva, como lo tiene que reconocer Martin en este pasaje: "We can, if we like, distinguish between "atomic" and "molecular" facts, but not independently of the given 
hasta abarcar toda la realidad, tal como lo hace Whitehead cuando escribe:

La naturaleza es un proceso.

Y, casi inmediatamente:

El proceso de la naturaleza puede denominarse también el paso de la naturaleza (35).

Munsat expresa la misma idea cuando afirma que:

The discovery and examination of the process is a discovery and examination of the principles according to which the physical world runs (36).

Es decir, el mundo físico -o el mundo, simplemente-, es un proceso.

Vemos, pues, que dentro de los límites establecidos por la aplicación del término - que, prácticamente, va de la unidad dinámica que hemos llamado hechó a la totalidad del ser, tal como lo acabamos de ver- tenemos una innúmera cantidad de procesos, el de una infección, el de una revolución, el de una elección, el - casi antonomástico- proceso judicial, el de la metamorfosis del sapo, el histórico, el biológico, etc., etc.

Ahora bien, retomandoelahiloprincipal de nuestra exposición, el punto fundamentalostablecidoi hasta aquí es la división -que nos sugiere la observación- de la realidad en cosas y hechos

language system-L" (Martin, Op. cit., p. 276). Y así tenemos que los pintorescos hechos negativos del no menos pintoresco lord inglés son, en realidad, proposiciones falsas de las que, en algún lugar, dice: "Suponer que haya en el mundo real de la naturaleza todo un conjunto de proposiciones falsas dando vueltas de un lado para otro resulta monstruoso para mi mentalidad. No puedo ni siquiera ponerme a suponerlo. No puedo creer que se den allí en el mismo sentido en que se dan Ios hechos" (Op. cit., p. 313). Quizá sea más mostruoso aún suponer que existen hechos negativos vagando de aquí para allá como las almas en pena de algunas mitologías ingenuas. Pero, volviendo a la afirmación de nuestro filósofo, también es monstruoso suponer proposiciones verdaderas dedicadas a tan poco provechosa actividad, aun cuando sus vagabundeos los realicen -o realizacen - fuera de los límites espaciotemporales de la realidad, tal como parece que lo supusiesen algunos filósofos. Cf. las dos últimas citas de la nota 31 .

(35) A. N. Whitehead. Op. cit., p. 66.

(36) Stanley Munsat: "What is a Process?". En American Philosophical Quarterly. Philadelphia, Vol. 6. 1969, p. 83. 
(37). Aún cliando la división en sí misma no es algo que exista en forma natural, objetiva, ni exacta; hemos visto que a todo lo que existe, es, está o hay se le puede aplicar la palabra 'cosa' y lo mismo podemos hacer con 'hecho'. Sin embargo, como también lo hemos visto, hay cierta diferencia que se puede apreciar en la observación de la realidad y que se expresa en la mencionada división entre ccsas y hechos.

Concretándonos a estos últimos hay varias cuestiones interesantes que pueden plantearse. La primera de ellas consiste en establecer si los hechos son o no son simples y qué se quiere decir con este último término. En general se sostiene que no son simples. Así Russell escribe:

Por "hecho" doy a entender algo complejo. Si el mundo no contiene ningún elemento simple, todo lo que contenga, entonces serán hechos; si contiene elementos simples, serón hechos todo lo que contenga con excepción de dichos elementos simples... (38).

En otro lugar afirma:

Hay particulares, cualidades y relaciones de diversos órdenes, toda una jerarquía de especies diferentes de simples [...] El único otro género de objetos con que nos encontramos en el mundo son los llamados hechos, que constituyen lo afirmado o negado en las proposiciones. $Y$ en modo alguno son realmente entidades en el mismo sentido en que lo son sus elementos constitutivos $(39)$.

Sobre este mismo puntorgodemos recordar la cita de Whitehead -Cf., supra, p. 4 de este trabajo- según la cual "la última sustancia de la naturaleza" son los hechos, o acontecimientos, como prefiere denominarlos. Pero, en otro lugar de la misma obra a la que pertenece el pasaje acabado de citar, también afirma que

el acontecimiento es un hecho complejo,...

(37) Que, en rigor, es la misma que hacen muchos filósofos, sea explícita o implícitamente; así, por ejemplo, Strawson señala que en una descripción de la realidad se puede hablar de "events and processes, states and conditions, on the one hand; and of material bodies or things posessing materials bodies on the other..." (Individuals. An Essay in Descriptive Metaphysics, New York, Anchor Books, 1963, p. 63). Evidentemente, al hablar de cosas que poseen cuerpos ya se está siendo más metafísico que descriptivo.

(38) Bertrand Russell. Op. cit., p. 400.

(39) Op. cit., p. 380 .

(40) A. N. Whitehead. Op. cit., p. 93. 
Ahora bien, si los hechos son complejos, ¿cuáles son los elementos simples que los componen? Russell señala que

las cosas, con sus cualidades o relaciones son, con toda evidencia, componentes, en uno u otro sentido, de los hechos en que dichas propiedades y relaciones entran en juego (41).

$Y$, en otro ensayo suyo:

Decir que los hechos son complejos es lo mismo que decir que tienen elementos constitutivos. Que Sócrates era griego, que se casó con Jantipa, que murió por beber la cicuta, son hechos que poseen algo en común, a saber que versan acerca de "Sócrates" del que se dice en consecuencia que es el elemento constitutivo de cada uno de ellos (42).

Tenemos, pues, que para Russell, los elementos constitutivos de los hechos son cosas, cualidades y relaciones. Pero, como estas dos últimas categorías de existentes no se dan, no existen en tanto no se dan o existen las cosas, llegamos al punto en el cual sólo podemos afirmar la existencia de las cosas, con las que y en las que se dan o existen las cualidades y entre las que establecemos las relaciones.

Por su parte, Whitehead señala que:

Un objeto es ingrediente del carácter de un acontecimiento.

Y, más adelante, Gơdloria que estảe

empleando elotérminocćingrésión"vpara significar la relación general de los objetos con los acontecimientos.

Y también que:

Un objeto es un ingrediente de todo cuanto le rodea, y lo que le rodea es indefinido (43).

Ahora bien, ¿qué son, para Whitehead, los objetos? En primer lugar, mencionemos que los clasifica en tres grupos, sensoriales, perceptibles y científicos. Y luego nos da la caracterización de cada uno de estos tres tipos:

Un objeto sensorial es un factor de la naturaleza propuesto por la toma de conciencia sensorial que (I), en la medida

(41) Bertrand Russell. Op. cit., p. 269.

(42) Op. cit., p. 400.

(43) A. N. Whitehead. Op. cit., pp. 161, 162 y 163. 
en que es un objeto no participa en el paso de la naturaleza, y (II), no es una relación entre otros factores de la naturaleza [...] Ejemplos [...] una especie particular de color, digamos el azul Cambridge; una clase particular de sonido, [...], o una especie particular de sensación (44). El objeto perceptivo es el resultado del hábito de la experiencia [...] Hay dos géneros de objetos perceptivos, a saber, "objetos perceptivos ilusorios" y "objetos físicos" (45). Los objetos físicos son los [...] que percibimos cuando nuestras sensaciones no son defraudadas, tales como sillas, mesas y árboles (46). Los objetos científicos [...] Encarnan aquellos aspectos del carácter de las situaciones de los objetos físicos que poseen una permanencia y son expresables sin referencia a una relación múltiple que incluya un elemento percipiente. Sus relaciones recíprocas están caracterizadas por una cierta simplicidad y uniformidad. Finalmente, las características de los objetos físicos observados y de los objetos sensorales pueden expresarse a base de esos objetos científicos [...]. Estos objetos científicos no son en sí mismos meras fórmulas de cálculo; porque las fórmulas han de referirse a las cosas de la naturaleza, siendo los objetos científicos las cosas de la naturaleza a las que se refieren las fórmulas (47).

Las citas anteriores, un poco más extensas -y continuasde lo usual, las hemos hecho con el ánimo de aclarar al máximo el esquema que Whiteheadotieneade la realidad o naturaleza. Y dicho esquema es el siguienteiccinelli Converso"

Hechos, o acontecimientos, conformados por:

Objetos, divididos en:

-Objetos sensoriales: cualidades.

-Objetos perceptivos, subdivididos en:

a) ilusiones, $y$

b) cosas: mesas, sillas, árboles, etc.

-Objetos científicos.

Preferimos el esquema menos abigarrado y - creemos- más natural de Russell. Hay hechos y estos hechos están conformados, integrados, constituidos, compuestos por las cosas -la otra cate-

(44) Op. cit., pp. 166-167.

(45) Op. cit., p. 173.

(46) Op. cit., p. 174 . Un poco más adelante (p. 189), agrega: "Un objeto material físico es un pedazo corriente de materia [...] objetos simples, tales como valores o sonidos los llamo objetos sensoriales".

(47) Op. cit., p. 176. 
goría en que se divide, o dividimos, la realidad-. Podemos ampliar lo anterior señalando que los hechos son el resultado -inevitable, si se quiere-, de la interacción de las cosas. $Y$, por supuesto, admitido el dinamismo inherente a la realidad, a la larga, todo es un hecho; $\mathrm{y}$, además, ocurre que así aplicamos el término cuando la ocasión lo requiere. La simple existencia de una cosa es, considerada desde esta perspectiva, un hecho, incluso si esa cosa es la pirámide o la esfinge. Pero, no olvidemos que la diferencia entre ambas categorías estriba, fundamentalmente -como se ha mencionado repetidamente-, en su temporalidad. Unas se dan a lo largo de un tiempo más o menos prolongado, los otros pasan, transcurren, ocurren, suceden, acontecen; aunque, por supuesto, los primeros, las cosas, también son temporales, pese a que algunos filósofos crean lo contrario. La pirámide es una cosa, un rayo o un accidente (48) es un hecho. El gol es un hecho, la pelota - sin la que no podríamos hablar del gol, no habría el gol-, es una cosa.

- Las cosas, a su vez, tampoco son simples, tienen partes. En el lugar oportuno vimos que cada parte puede convertirse en una cosa independiente, pero esto sólo sucede dentro -o hasta- ciertos límites, aún cuando éstos no sean ni rigurosos, ni naturales, ni objetivos. Desde otro ángulo, las cosas están constituidas por cualidades o propiedades; con esto queremos significar que cualquier cosa tiene - por lo menos- cierta extensión y cierto peso y que no existe nada que no posea dichas características y sea una cosa.

Vemos, pues, que las cosas son los elementos constitutivos de los hechos. (Las propiedades entanto que se dan en las cosas, no por derecho propio; lo que hay en la realidad son las cosas $y$, claro está, las cosas no solamente poseen cualidades, sino que es imposible que existan sin ellas. Con las relaciones se da una situación análoga, aunque más precaria). Repitiendo la formulación que creemos la más adecuada, podemos decir que los hechos son el producto de la interacción de las cosas entre sí.

La segunda cuestión que puede plantearse con relación a los hechos es la de si éstos tienen alguna estructura, algún orden interno que permita su comprensión racional. La respuesta parece ser afirmativa. Martin escribe:

Facts have an internal structure. In them objects are "com-

(48) Un atropello es un acontecimiento; en cambio, el obelisco es un objeto, porque es "una corriente continua, limitada de acontecimientos tales que un pedazo de esa corriente durante una hora, un día o un segundo, tiene el carácter de ser la situación del obelisco" (Op. cit., p. 186). El término 'objeto' es empleado por Whitehead, principalmente, como sinónimo de cosa; aunque le da un sentido más amplio; cf. supra, p. 19. 
bined in a definite way", this way being "the.structure of the [...] fact" (49).

Y esto se toca muy de cerca con el último punto que - con referencia a los hechos- queremos tratar y que es el de la relación - o falta de ella- entre los hechos. Así como las cosas - cuya interacción produce los hechos-guardan entre sí cierto orden, conforman cierta estructura, que es la del hecho o, simplemente, el hecho, ocurre que los hechos también se estructuran entre sí. Como dice Whitehead:

Los hechos concretos de la naturaleza son acontecimientos que muestran una determinada estructura en sus relaciones recíprocas y ciertas características por su cuenta [...] las relaciones estructurales recíprocas entre los acontecimientos son tanto espaciales como temporales (50).

$Y$ no es solamente que los hechos, la totalidad de ellos o realidad, estén interrelacionados sino que, además, son -en mayor o menor grado - interdependientes. Adoptando una conocida imagen, podemos describir la realidad como una enorme red en la cual a cada instante van apareciendo innumerables nudos -cada uno de ellos producto de una infinita gama de posibilidades-i y en la cual, evidentemente, todos los nudos están relacionados entre sí y son interdependientes.

Esta interrelación entre los hechos que constituyen la realidad -que a una primeray y superficiabojeada podría aparecer como un confuso montón de particularidades desordenadas, inconexas y caóticas-, ha sido una de las más antiguas intuiciones de la humanidad, y su búsqueda uno de los ejes en torno a los cuales ha girado la historia o, más bien, si la metáfora no estuviese ya demasiado manoseada, -o pisoteada, cuando la manejan quienes carecen de manos- uno de los motores de la historia, y sus conquistas, los avances logrados por dicho motor cuando funciona, es decir, cuando encuentra quien sepa hacerlo funcionar (51).

(49) Martin. Op. cit., p. 276.

(50) A. N. Whitehead. Op. cit., p. 187.

(51) George Sarton. Seis alas. Buenos Aires, Editorial Universitaria de Buenos Aires, 1960, pp. 215 y 14:

Como el núcleo de la historia es el progreso del conocimiento, la historia de la ciencia ha de constituir el núcleo de la historia en general [...] si deseáramos exponer el progreso de la Humanidad, la historia de la ciencia constituiría el verdadero eje de nuestra exposición. La historia de la ciencia proporciona el eje o, digámoslo así, la armazón de.la historia de la Humanidad. 
Que la tarea de la ciencia es, fundamentalmente, buscar y hallar dichas interrelaciones lo dice Whitehead con su claridad y sencillez habituales:

En Ciencias Naturales, "explicar" significa meramente descubrir "interconexiones" (52).

Descubiertas las interconexiones obtendremos una imagen clara y armoniosa, es decir, racional, de la realidad. A ello aspiraron los $\phi \iota \lambda o \sigma o \theta$ o de Thales a Aristóteles, reconociendo sus limitaciones e ignorancia; ésa fue una de las débiles pero esperanzadoras lucecitas que brillaron tenuemente en los conventos medievales; fue la meta estimulante del pensador renacentista; el sueño que creyó casi logrado el científico de la época moderna. Hoy sabemos que la tarea no tiene término; pero quienes han dedicado sus vidas, su talento y sus energías a la construcción de dicha imagen, la imagen científica del mundo, son los mayores benefactores de la humanidad.

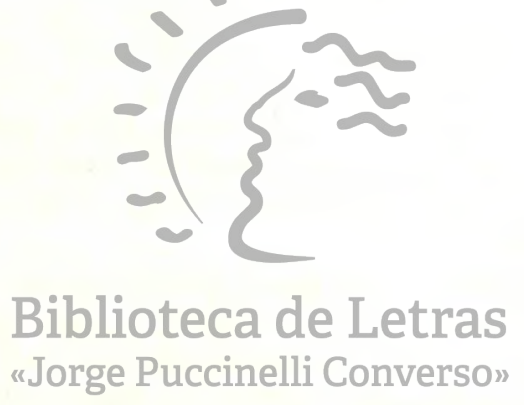

(52) A. N. Whitehead. Op. cit., p. 112. 